\title{
Digital domes that become urban symbionts
}

\author{
Fernando García Amen \\ Universidad de la República, Uruguay \\ efe@fadu.edu.uy \\ Rodrigo Martín Iglesias \\ Universidad de Buenos Aires, Argentina \\ rodrigo.martin.iglesias@gmail.com
}

\author{
Alejandro Schieda \\ Universidad de Palermo, Argentina \\ aschieda@nn-ar.com
}

\author{
Federico Lagomarsino \\ Universidad de la República, Uruguay \\ lagomarsino.federico@gmail.com \\ Santiago Miret \\ Universidad de Buenos Aires, Argentina \\ smiret@gmail.com
}

\begin{abstract}
Montevideo has a new visitor. In the heritage building of the german architect Karl Trambauer, located in the Old City, a new presence was installed filling the vacuum left by its former collapsed dome, seeking to restore a message, adding a new vision and recovering the lost dialogue between the architecture, the city and its inhabitants. This paper summarizes and explains the experience of the workshop Adaptation 2015, held on September 2015 at the Universidad de la República, Uruguay. Exposing the theoretical framework, design strategies, morphogenetic development, digital manufacturing experimentations, conclusions and open questions from the experience made. We will go through this temporary intervention on Trambauer's building, being a rare but symbiotic object, with parametric genes, digital and handcrafted manufacture, and also looking for the impact of theory and academic practices in the city.
\end{abstract}

Keywords: Urban intervention - Cities - Heritage - Parametric design - Digital fabrication

\section{Introduction}

During the nineties some architects (at the time considered "outsiders") started a research path involving digital tools to arise the nascent paradigm of non-linear processes of the emergent General System Theory developed in the thirties. Today the availability of open source programming, modeling and manufacturing software makes it possible to shorten the gap with those early produced systems.

Nevertheless, these processes often lack from disciplinary foundations involving architecture as a vector of a systematic event. In this way, the artifacts, installations and buildings produced share a lack of questioning of the status quo, becoming barely propositive and unable to reach innovative instances of the process. The image plays a fundamental role in these processes (that we could call "procedural technicisms") while the technique is overrun by images leaning towards mimetic approaches to standard and classic beauty parameters such as harmony, proportion and symmetry.

The current generation of parametric-generative software allows us to access complex generation systems with relative ease reinforcing this condition of image above thesis. The gap between the original theory of systems and the new visualization methods of complex systems through specialized software should be filled with the problematization of these systems in relation to (in our case) the architectural discipline, in order to escape from the banal production of images typical of the marketing and advertisement worlds and move towards the production of knowledge in association with a shift in the current status quo of Architecture.
Following this line, a group of researchers, architects and designers from the Universidad de la República (Uruguay), the Universidad de Buenos Aires and the Universidad de Palermo (Argentina) have developed a research project that aims to reproduce some parasitic generative techniques that will be tested in the digital production of a specific tectonic artifact. In the heritage building of the german architect Karl Trambauer, located in the Old City of Montevideo, a new presence was installed filling the vacuum left by its former collapsed dome, seeking to restore a message, adding a new vision and recovering the lost dialogue between the architecture, the city and its inhabitants. We will go through this temporary intervention on Trambauer's building, being a rare but symbiotic object, with parametric genes, digital and handcrafted manufacture, and also looking for the impact of theory and academic practices in the city. This artifact tries to reinterpret a product of classical architecture, specifically the dome as way to crown a building on a street intersection. Domes have a long tradition in architecture as fundamental elements of a time where the previously named qualities (harmony, symmetry, rhythm, proportion) were conducted and translated into tectonic elements. The dome is the characteristic element in classic composition methods, reaching its highest point with the Roman Pantheon, a symbol of classic composition.

In this sense, taking a classic dome as the object of intervention and reconfiguration has a deeply disciplinary interest and looks to open a debate around an emblematic object of the architectural tradition through the use of generative digital tools in order to connect aspects deeply related to contemporary technique with classical notions, 
moving away from preconceptions associated to norms of traditional beauty.

This artifact will become a social attractor and intent of reflection on the historical buildings and urban heritage. A contemporary artifact with a classical theme, reinterpreted, subverted, transformed. This subversion aims to put in practice experimental disciplinary aspects currently being questioned from a visual standpoint. The focus will be set on how to articulate critical-historical issues with the idea of the dome and the specific technique in relation to generative processes associated to complex systems.

\section{Homo Faber Revolution}

There is a traditional separation between the project (design process) and the construction work (production) that can be traced to the origins of the architecture as a discipline. In $D e$ Re Aedificatoria, Leon Battista Alberti (1450) said that "architecture is composed of two parts, the Lineamenta (derived from the mind) and the Matter (derived from nature) mediated by the skilled craftsman". This implies that there is a process of communication between the two parts involved, so there are media and languages (representations) that make this possible, but also implies that architects are losing contact with materials and building processes. Robin Evans (1995) describes how this has led to a condition in which architects work under a peculiar disadvantage they do not work directly with the object of his thinking, they always work on it through some medium intervention. The elimination of waiting to be animated by a mobile point of view. In their search for systems that can simulate the appearance of life, industry special effects and animation it has developed a set of useful tools for these investigations; as animation software using a combination of deformable surfaces and physical forces. Because of the convergence of technological processes and computer models of biological growth, development and transformation can be investigated using animation rather than conventional design software architecture attended. Instead of being designed as stationary inert forms, space is plastic, flexible and mutable in its dynamic evolution through movement and transformation. In simulations animation, the way is not only defined by the internal parameters, as is also done by a set of other external, invisible and gradients fluctuating forces, including: gravity, wind, turbulence, magnetism and swarms of particles moving.

In the foreword to his pivotal book Architecture in the Digital Age: Design and Manufacturing (2003), Branko Kolarevic claims that "one of the deepest aspects of contemporary architecture is not the rediscovery of complex curved shapes, but the new ability to generate construction information directly from the design information through new processes and techniques of digital design and production". Though, It is believed that a greater degree of control over design and production information, along with customizable file-to-factory processes, will facilitate a range of new spatial, formal, performative and methodological possibilities in architecture. While these ideas seem reasonable in theory and have been

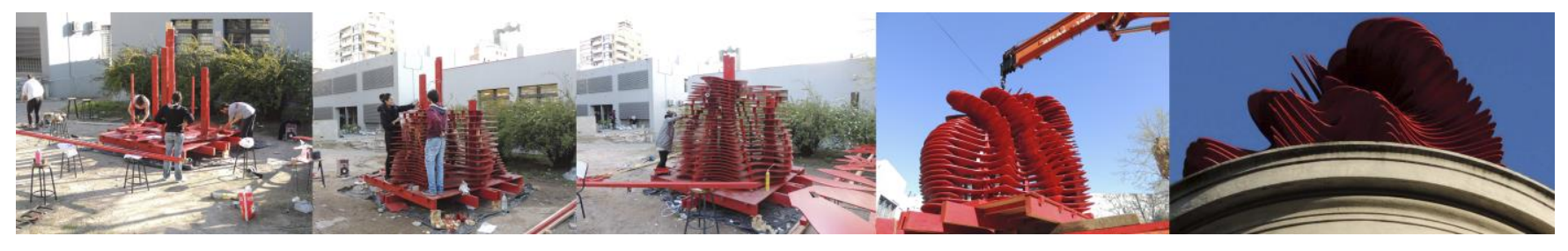

Figure 1: Assembling process in all stages

the architect of the construction of the building itself introduces a discontinuity in the process of design and construction. This discontinuity had many benefits but also introduced problems. On one hand, the potential miscommunication and misinterpretation of information, and on the other, the increasing abstraction of the experience of architects. Ceccato (1999) suggests that a gradual erosion of practical and material knowledge among architects has resulted in "the figure of the emancipated designer ... whose work remains pure and detached from mundane cares of manufacturing and construction."

According to Greg Lynn (1995), architecture, both in its conception and its realization has been understood as something static, fixed, ideal and inert. Issues of movement and dynamic architecture are often treated as pictorial views of static forms. Not only the buildings have been constructed as static forms, but the most important thing is that the architecture has been conceived and designed based on models of stasis and balance. Typically, the computer animation software reinforces this assumption that architectural design belongs to the static Cartesian space partially implemented in practice, the extent to which this territory can be explored and potentially expanded is currently limited. The reason for this is that the commercially available design software typically allows constructing the geometry independently of the material and structural considerations. Kilian (2004) said that this situation often leads to a sculptural design process where the translation of the form in buildable components develops after establishing the form.

At the same time, we are faced with the revolution of making, or rather, a technological insurgency of the well-known culture of do-it-yourself. Traditional fields such as blacksmithing and carpentry are hybridized by electronics, robotics and $3 \mathrm{D}$ printing. But like everything to come, it is already here. The maker culture emphasizes peer learning (informal networking and learning) of practical skills to apply creatively, from a stethoscope built from a smartphone, a doorbell capable of recognizing faces or a biodegradable tableware made of lactic acid. Imagination comes back to power, but this time accompanied by new technologies and intense desire to experiment and make prototypes. Platforms for new ventures like Kickstarter are helping these "makers" 
to find funding for their initiatives and the Maker Faires are must for anyone who wants to know what is truly innovative. Perhaps the most innovative of all is not purely technological, or the possibilities for the future that suggests, but the possibility that we stop being mere consumers to be "makers" and that is truly revolutionary.

\section{Parasites y Symbionts}

In the context of an intervention of a heritage listed building, mixed with the design experimentation using parametric morphogenesis and digital fabrication, we develop a strategy to cope with complexity and face the challenge. As we said before, biological growth and its computer models can be used to conceive new kind of forms or new relationships between forms. For that we choose two biological design analogies, the parasite and the symbiont. Parasitism is a nonmutual symbiotic relationship between species, where one species, the parasite, benefits at the expense of the other, the host. Unlike predators, parasites typically do not kill their host, are generally much smaller than their host, and will often live in or on their host for an extended period. In the process of parasitism, the species that carries out the process is called parasite or guest and the parasitized species is called host.

Parasites that live inside the host or host organism are called endoparasites and those living outside are called ectoparasites. The mesoparasites have a part of their body facing outward and the other anchored deeply into the tissues of its host. A parasite that kills the organism where he is staying is called parasitoid. As every parasite remains an organism, it may be converted in turn into a third species host. The parasite that parasitize other parasite is often referred as hyperparasite, an example of this are the satellite viruses, which require other virus to reproduce.

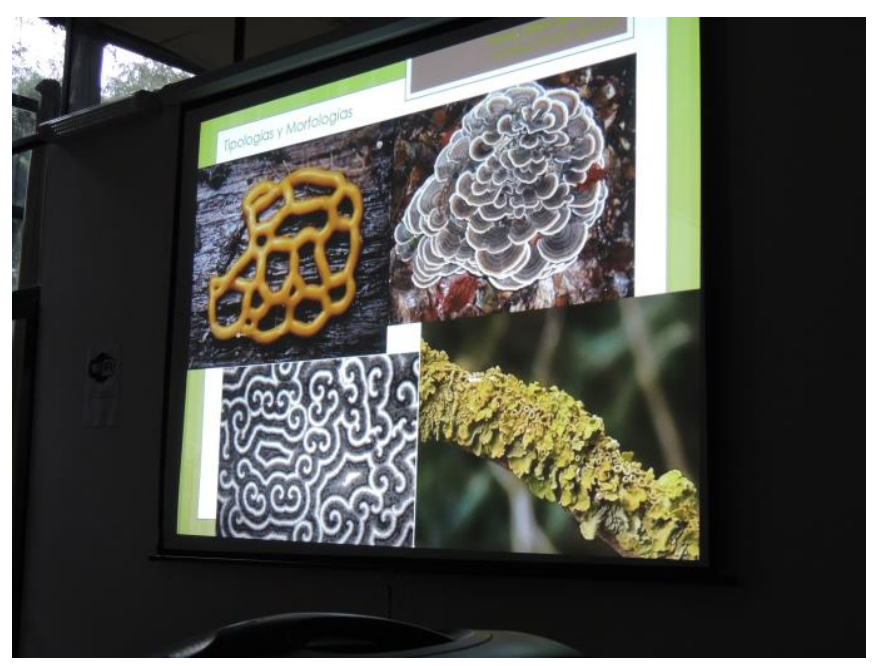

Figure 2: Theoretical conceptualization in class.

Another feature of parasitism is that although the parasite must adapt to the immune response and, in general, parasitic life, the host also must do. This is because the parasitic population exerts selective pressure on it, so that guest or parasite and host simultaneously co-evolve as a result of parasitism. Many parasites, particularly microorganisms, are evolutionarily adapted to particular host species; in such interactions the two species have evolved each on its side within a relatively stable relationship that does not kill the host quickly (which would also be detrimental to the parasite). In some cases, the relationship with their host parasite may be closer, and even get to form a co-speciation among them.

On the other hand, the term symbiosis (from Greek oúv "together" and ßíwoıs "living") applies to biological interaction, the close and continuing relationship between organisms of different species. The organisms involved in the symbiosis are called symbionts. "Symbiosis, the union of different organisms to form new groups, has proven to be the most important force for change on Earth" (Lynn Margulis, 1989). Therefore, after several explorations and debates, we decided to take the Symbiont analogy to use as a morphogenetic strategy for the last part of the workshop with the students, which results in a biological reinterpretation of the original dome and a design process that focuses on the interaction between the existent building and the new form. Thereby, the designer builds a generative system of formal production, controls their behavior over time, and selects the forms that emerge from its operation, following the morphogenetic strategy, instead of a mere aesthetic and plastic preference (Kolarevic, 2003).

\section{Methodological procedures}

The Workshop that prompted this research is part of a platform named Adaptation, which was hosted at the University of the Republic in Montevideo, Uruguay with students, architects, artists, companies, technicians and instructors of the same university and also from the University of Buenos Aires and the University of Palermo, both from Buenos Aires Argentina. A heterogeneous team was put together in order to move away from local parameters associated to beauty and tradition.

\section{The process consisted of three stages:}

1. Development of the theoretical concept and discussion of methodological and technical aspects depending on the nonlinearity-parasite relationship. This first stage focused on the conceptual development of the Digital Paradigm associated with issues of parasitic relations to build ideas between complex generative processes and parasitic behavior on pre-existent systems. Because the intervention would take place on an existing building of Montevideo's Ciudad Vieja, the proposal would have to address preexisting links and relationships, understanding them as systems. In this sense the new dome is not indifferent to its host and is able to develop links and internal feedback loops operating as a physical configuration sensitive to its specific placement.

The issue of reconceptualization of the classic dome was addressed from the point of view of the form and its tectonic qualities over functional or aesthetical notions that basically elaborate on preconceived notions. In this way, possibilities of formal variation associated to the traditional dome were 
explored, using the original (now demolished) dome of the building.

2. Testing and selection of the generative system. How do you create form suspending the variables of function and aesthetics? The first tests involved models of traditional domes, which were affected based on a sequence of cross sections. Variations in the repetitive process of form generation were introduced, small alterations in the generation patterns to slightly interrupt the lineal descent of gravitational forces through the dome shell. These initial tests developed into a dome shape, whose generatrixes shift, rotate and set back according to variations in the generative pattern. The traditional dome is distorted but the shape is preserved. The same formal logic that builds the traditional dome is used to rebuild this new artifact.

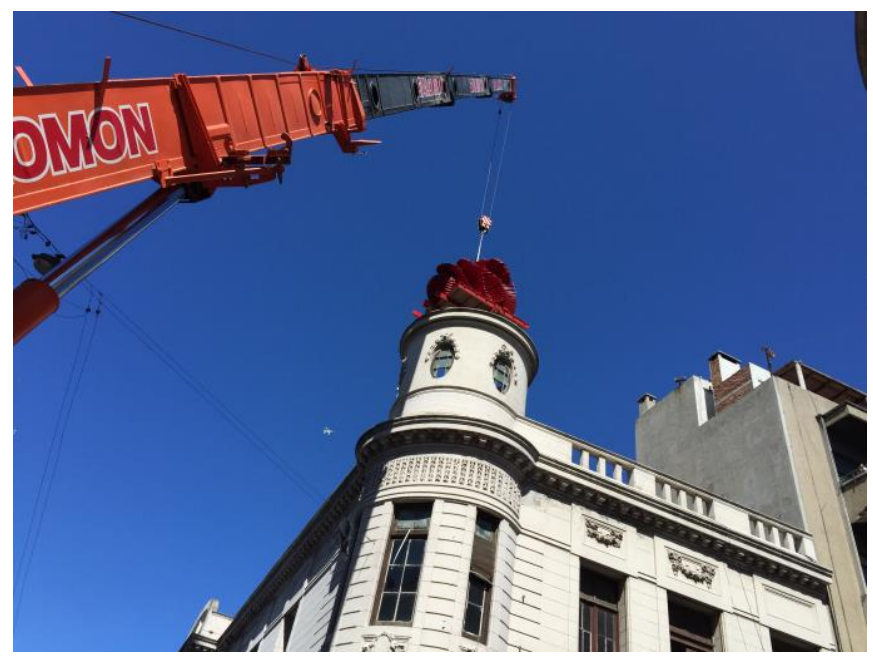

Figure 3: Mounting the dome in site.

3. Fabrication and installation of the device in place. Finally, the object is fabricated following the logic of its formal generation. The affected generatrixes for the parasitic dome are the ones to be machined and assembled to rebuild the form.

\section{Results}

The device in question was based on the reformulation of a dome of the XX Century (now demolished) in the Old City of Montevideo, Uruguay. The manufacturing process was carried out by a CNC router at the University. The installation of the device in place was conducted by a major logistics.

The new citizen of Montevideo's Old City is new and old at the same time. It contains in itself the classical dome, its tectonics and generative form, its directrix and generatrixes while proposing a new way to consolidate the top of the classical building. It clearly responds to a contemporary aesthetic parameter, which emerges not from a mimetic position towards an imaginary zeitgeist but as the result of a generative logic imbued with notions of the classical dome. It proposes, at the same time, a new use. A function related to the social, to the community and the urban transformation.

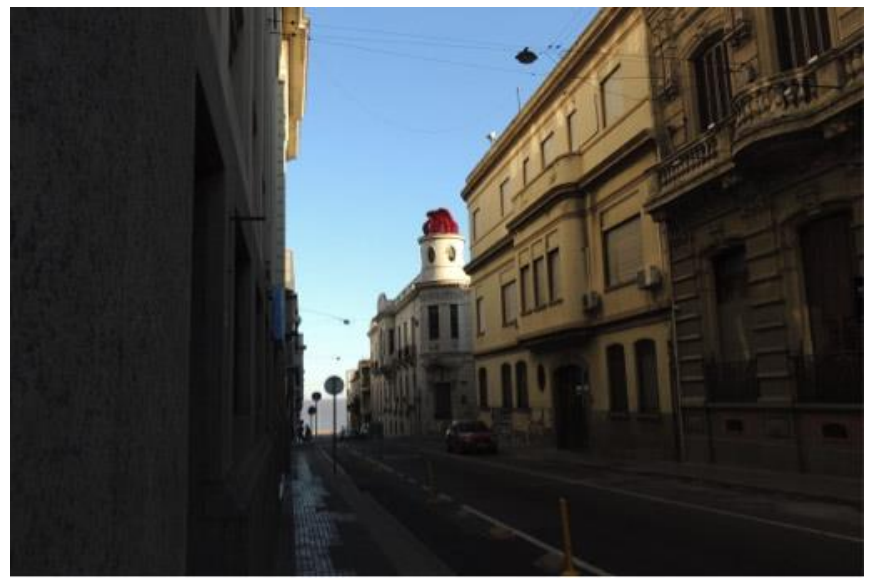

Figure 4: Historical building the new dome.

\section{Discussion}

This research aims to raise the discussion in two levels:

1. Digital generative processes often tend to behave autonomously with material for materialization (manufacturing), therefore it is necessary to call for the development of mixed protocols or procedural feedback that would serve the purposes of testing models and materials.

2. The historical reformulation of an architectural system (the dome), not its functionality or aesthetics, but their mode of production (intellectual and material) and its historical relevance to the Zeitgeist in extending its life.

Are there ideal digital protocols in terms of their manufacture? Is it possible to generate these links of processual feedback with the technology that we have? What is the critical relevance of these models in relation to contemporary culture confronted with an urban environment whose history is embodied?

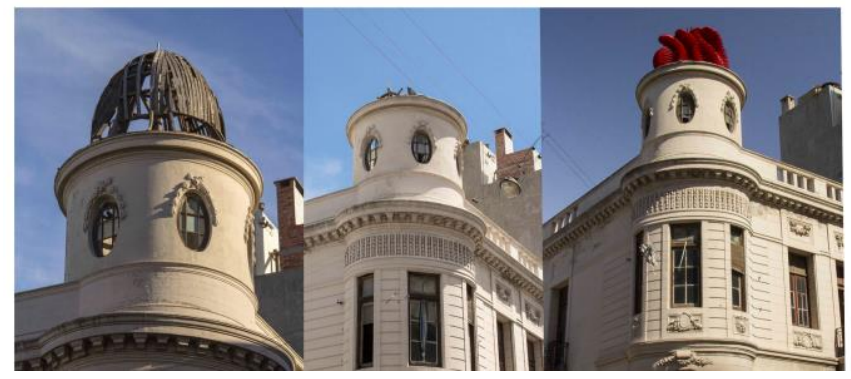

Figure 5: Sequence year 2011, 2012, 2015.

\section{Aknowledgments}

Professors: Marcelo Payssé, Paulo Pereyra, Luis Flores, Juan Pablo Portillo, and Gabriela Muniz.

Consultants: Alfredo Ghierra, Fernando Foglino, and Ignacio Silva. 
Students: Santiago Páez Revuelta, Elvis Marrero, Luis Blau, Juliana Mansulino, Lucia Sosa, Lucia Borche, Lucía Lin, Lucía De Benedetti Trobo, Mónica Cervieri, Gabriela Barber, Leandro Villalba, Tania Pérez Mesones, Matias Yañez, Simone Cammilletti, Mara Márquez, and Giannina Carabelli.

Companies involved: Barraca Parana, Barraca La Chacarita, Carpintería Valsain, Fabrix, Maderas de La Franja, Antrix, Stiler, Salomón Grúas, Cámara de Construcción de Uruguay, Kosak, Nalon, Copiser, Pocitos Hostel, BH Rent, Bysic.

\section{References}

ALBERTI, L. B. “De re aedificatoria $(1450,1485)$ ", en Opere volgari. Vol.III, Bari: Laterza. 1973.

CARPO, Mario. The Digital Turn in Architecture 1992 - 2012. London: John Wiley \& Sons, 2013.

CECCATO, C. "Microgenesis. The Architect as Toolmaker: Computer-Based GenerativeDesign Tools and Methods", in: Soddu,
C. (ed.): The Proceedings of the First International Generative Art Conference. Generative Design Lab at DiAP, Politecnico di Milano University. 1999.

EVANS, R. The Projective Cast. Architecture and its three Geometries, Cambridge: MIT Press. 1995.

FRAZER, John. An Evolutionary Architecture. London: AA Publications, 1995.

KILIAN, A., Ochsendorf, J.O., Particle-spring systems for structural form finding, in: Journal Of The International Association For Shell And Spatial Structures: IASS, Vol. 46, pp. 77-84, 2005.

KOLAREVIC, Branko, ed. Architecture in the Digital Age: Design and Manufacturing. New York \& London: Spon Press - Taylor \& Francis Group, 2003

ORTEGA, Lluis. Digitization takes over. Barcelona. RotoVision, 2009.

Please delete this line after your work is complete. It is just a reference to maintain the graphic balance of the two columns. 